\title{
Does Universal Test and Treat Strategy has an effect on Antiretroviral Therapy Adherence? A Comparative Cross-sectional Study
}

Yitayish Damtie ( $\sim$ yitutile@gmail.com )

Wollo University

Dabere Nigatu

Bahir Dar University

Fentaw Tadese

Wollo University

Melaku Yalew

Wollo University

Research article

Keywords: Antiretroviral therapy, Adherence, HIV/AIDS, Ethiopia

Posted Date: August 29th, 2019

DOI: https://doi.org/10.21203/rs.2.13576/v1

License: (c) (i) This work is licensed under a Creative Commons Attribution 4.0 International License.

Read Full License 


\section{Abstract}

Background : Poor adherence is a critical problem in managing Human Immunodeficiency Virus (HIV ) infected patients receiving Antiretroviral Therapy (ART). Evidence of adherence to antiretroviral therapy after initiation of Universal Test and Treat (UTT) strategy was limited in Ethiopia. Hence, this study aimed to compare adherence to antiretroviral therapy before and after the initiation of universal test and treat strategy, including factors affecting adherence among HIV positive adults in Dessie town.

Methods: A comparative cross-sectional study was conducted on 594 HIV positive adults selected using consecutive sampling. Interview and patient record review were used to collect data. The data were analyzed using SPSS version 23. Bi-variable and multivariable logistic regression model were used to identify factors associated with ART adherence. Adjusted Odds Ratio (AOR) with 95\% Confidence Interval (Cl) was used as a measure of association. Statistical significance was declared at a P - value less than 0.05 .

Result : The overall proportion of ART adherence using Morisky scale and self-reports were $52.3 \%, 95 \% \mathrm{Cl}$ : (48.4\%, 56.2\%) and 95\%, 95\% Cl: (93.5\%, 96.8\%) respectively. Absence of depression (AOR $=3.87,95 \% \mathrm{Cl}$ : $(1.96,7.64))$, eating three or more meals per day $(A O R=2.65,95 \% \mathrm{Cl}:(1.08,6.49))$ and absence of concomitant illness $(A O R=0.42,95 \% \mathrm{Cl}:(0.23,0.76)$ ) were significantly associated with better ART adherence.

Conclusion: The overall proportion of ART adherence measured by Morisky scale was very low while the proportion of ART adherence measured by self-report was high and consistent with the current World Health Organization (WHO) recommendation. Adherence to ART was not affected by the introduction of the UTT strategy in HIV treatment and care program. Depression, meal frequency and concomitant illness were factors associated with ART adherence. Efforts should be made to improve adherence through tailored interventions to overcome factors linked with poor adherence. Key words: Antiretroviral therapy, Adherence, HIV/AIDS, Ethiopia

\section{Introduction}

HIV/AIDS becomes the major threat to the world population. Although significant progress was seen in expanding HIV prevention and care services worldwide, 36.7 million people were living with HIV, 1.8 million people were newly infected and 1 million were died due to AIDS in 2016. Sub-Saharan Africa (SSA) shares $64 \%$ of new HIV infection (1). According to 2017 HIV related estimate, 722, 248 people were living with HIV, 22,827 people were newly infected and 14,872 were died in Ethiopia (2).

The WHO recommends a greater than $95 \%$ adherence to get the best outcome of antiretroviral treatment (3). But, suboptimal adherence became the major critical problems in managing HIV-infected patients receiving ART. Results of a meta-analysis in SSA indicated that a pooled estimate of only $55 \%$ of the populations achieved adequate levels of adherence (4). Evidence from a systematic review of adherence 
to ART in SSA also indicated that the average ART adherence score was $72.9 \%$ (5). In Ethiopia adherence to ART ranged from $63.8 \%-95.5 \%(6-10)$, most of them were below the WHO recommendation.

Sub-optimal adherence imposed a significant impact on the patient's health. It resulted in the development of detectable viral loads, declining Cluster Differentiation 4 (CD4) counts and disease progression $(11,12)$. For instance, result of longitudinal study showed that $0 \%, 8 \%$ and $41 \%$ of patient with $>90 \%, 51-90 \%, \leq 50 \%$ adherence were progressed to AIDS over the 13 months follow-up period respectively (13).A study also demonstrated that subjects with adherence levels of $0-79 \%, 80-99 \%$ and $100 \%$ had 53 cells $/ \mu \mathrm{L}, 159$ cells $/ \mu \mathrm{L}$ and 179 cells/ $\mu \mathrm{LCD} 4$ increment from the baseline to 12 months respectively (14).As studies have shown, socio-demographic factors(15), medication-related factors, health system factors(16) and psychosocial factors(5) were known to affect adherence to ART medication.

Previously, in 2013, WHO strongly recommended initiating ART among all HIV positive individuals with a CD4 count of 500 cells/mm3 or less by giving priority to those patients who had advanced HIV clinical disease (WHO clinical stage 3 or 4 ) or a CD4 count of $<350$ cells $/ \mathrm{mm}^{3}$ (17). But in 2015, WHO launched a new UTT strategy that involves early identification of all HIV-infected individuals, followed by immediate ART initiation regardless of CD4 count or Viral Load (VL). This will reduce the incidence of HIV infection and opportunistic infection, early disease progression, and the burden on the health service (18).

Ethiopian government adopted this approach in 2016 and began to implement it in ART clinics of Dessie Town since June 12 2016.

Even though adherence to ART had been well investigated before the initiation of universal test and treat strategy, only a little evidence exists about adherence after initiation of the UTT strategy. On the other hand, most of the studies used a single ART adherence measurement scale. So, the objective of this study was to compare antiretroviral therapy adherence before and after initiation of test and treat strategy and associated factors among HIV positive people who are attending ART clinics of Dessie town using multiple ART drug adherence measurements.

\section{Methods}

\section{Study Area, Study Design and Participants}

A comparative cross-sectional study was conducted in ART clinics of Dessie town from April15-June 5, 2018. Dessie is the center of South Wollo Zone located $401 \mathrm{~km}$ away from Addis Ababa, the capital city of Ethiopia and $480 \mathrm{~km}$ away from Bahir Dar. In Dessie town, two government hospitals and three health centers were providing ART service for 8,821 adult people enrolled before initiation of UTT strategy and 926 adult people enrolled after initiation of UTT strategy (19). The study population was consecutively selected HIV positive adults who were attending ART clinics indicia Town. Those HIV positive people aged greater than or equal to 18 years and who were on ART for at least three months were included in the study. 


\section{Sample size and Sampling procedure}

The sample size was calculated using Epi Info version 7.1 with the assumption of $89.1 \%$ ART adherence among people who didn't encounter opportunistic infection and 2.81odds ratio taken from a study done in eastern Ethiopia (20), 1 to 1 ratio of exposed to unexposed, $80 \%$ power and $95 \%$ confidence level. Thus, adding $10 \%$ for non-response, the minimum required sample size was 594 (297 for who started before UTT strategy and 297 for who started after UTT strategy). The sample size was proportionately allocated to each health facility providing ART service based on the average number of client flow per month. Then, the study participants were selected in order of their arrival until reaching the sample size.

\section{Data Collection Procedures and Measurements}

Data were collected by face to face interview using a structured and pretested Amharic version questionnaire. Medical records were also reviewed to identify clinical markers like CD4 count, viral load and WHO clinical staging. Five trained nurses have collected the data with supportive supervision of the principal investigator and one supervisor. The questionnaire composed of socio-demographic factors, medication-related factors, health care related factors, disease characteristics and psychosocial and behavioral factors that have taken from similar literatures $(5,15,16)$.

Patient Health Questionnaire (PHQ-9) checklist was used to assess depression. This checklist has nine questions with four response option taking a score between 0 and $3(0=$ not at all, $1=$ several days, $2=$ more than half a day and $3=$ nearly every day). Then, patients who scored greater than or equal to the median value were classified as having depression and patients who scored less than the median value were classified as have no depression (7). Ten questions with yes or no option were used to measure patients' knowledge to ART. If the patient gets the right answer coded as 1 if not coded as 0 . Then patients who scored greater than or equal to the median value were classified as having good knowledge whereas patients who scored less than the median value were classified as having poor knowledge (21). We had ten questions to assess patients' perceived stigma. The questions were with yes or no response options. The correct responses were coded 1 while the incorrect responses were coded 0 .Then patients who scored above the median value were considered as they had perceived stigma whereas patients who scored less than or equal to the median value were taken as they had no perceived stigma (22).Adherence to ART regimen was assessed by using two medication adherence measurement approaches: a sevenday patient self-report and eight items Morisky Medication Adherence Scales (MMAS). In, a seven-day patient self-report method: a patient is asked a question "did you miss in taking your ART drug in the last seven days?"If a patient answered 'yes' to this question classified as good adherence otherwise taken as poor adherence (20).In, the MMAS approach: a patient is asked eight questions designed to assess adherence to medication. Seven of the questions were with yes or no response options coded each as 1 or 0 respectively. The $8^{\text {th }}$ question was with five response options taking a score between 0 and 4 ( 0 always, 1 usually, 2 sometimes, 3 once a while and 4 never). Then, those patients who score less than or 
equal to the median value were classified as poor adherence whereas those patients who score greater than the median value were classified as good adherence (23).

\section{Statistical Analysis}

Data were coded and entered to Epi Data Version 3.1 and exported to the Statistical Package for Social Science (SPSS) Version 23 statistical software for statistical analysis. Descriptive statistics such as frequency, proportion, mean with Standard Deviation (SD) and median with Inter-Quartile Range (IQR) were computed. The association between independent variables and adherence to ART was made using a binary logistic regression model. All independent variables having a p-value less than 0.2 were included in the multivariable logistic regression model. Multicollinearity was checked using the Standard Error (SE). The p-value of the Hosmer and Lemeshow test of the model fitness was 0.87 . Variables having adjusted odds ratio with $95 \%$ confidence interval not inclusive of one was considered as statistically significant predictors of ART adherence.

\section{Results}

\section{Socio-demographic Characteristics}

In this study, 289 HIV positive adult patients who were enrolled before and 292 HIV positive adult patients who were enrolled after initiation of UTT strategy were involved and making a response rate of $97.8 \%$. The mean age of patients who were enrolled before and after initiation of UTT strategy was 36.2 with \pm $9.8 \mathrm{SD}$ and 36.7 with $\pm 8.6 \mathrm{SD}$ respectively. One hundred seventy-one $(59.2 \%)$ of patients who were enrolled before and $152(52.1 \%)$ of patients who were enrolled after initiation of UTT strategy were females. Two hundred thirty two $(80.3 \%)$ patients who were enrolled before and $218(74.7 \%)$ of patients who were enrolled after initiation of UTT strategy were urban residents. In case of marital status, 155 (53.6\%) and $178(61.0 \%)$ of patients enrolled before and after initiation of UTT strategy were married (Table 1).

\section{Psychosocial and Behavioral Characteristics}

Two hundred seventy eight (96.2\%) HIV positive patients who were started ART before and 285 (97.6\%) of patients who were started ART after initiation of UTT strategy used reminders to take their ART medication. Two hundred ten (72.7\%) patients who initiated ART before and 189 (64.7\%) of patients who initiated ART UTT strategy had good knowledge to HIV/AIDS and its treatment. Two hundred eighty nine (100\%) patients who initiated ART before and 291 (99.7\%) of patients who initiated ART after initiation of UTT strategy took adherence counseling. Two hundred sixty four (91.3\%) and 270 (92.5\%) of patients enrolled before and after initiation of UTT strategy disclosed their HIV status to other individuals respectively. Fourteen (13.8\%) patients who initiated ART before and $35(12 \%)$ of patients who initiated ART after initiation of UTT strategy experienced depression (Table 2). 


\section{Diseases related Characteristics}

A viral load test was done for all patients who were enrolled before the initiation of UTT strategy. As a result, $247(85.5 \%)$ and $42(14.5 \%)$ patients had a viral load of $<1000 \mathrm{copies} / \mathrm{ml}$ and $\geq 1000 \mathrm{copies} / \mathrm{ml}$ respectively. One hundred ten (37.7\%) patients who started ART after UTT strategy had no viral load test result, $156(53.4 \%)$ patients had a viral load of $<1000 \mathrm{copies} / \mathrm{ml}$ and $26(8.9 \%)$ patients had a viral load of $\geq 1000$ copies/ml respectively. One hundred fifty-six (54\%) patients who started ART before and 65 (22.3\%) patients who started ART after UTT strategy had a baseline CD4 count of $<200 \mathrm{~mm}^{3}$. Forty-four (15.2\%) patients enrolled before-and 237 (81.2\%) patients enrolled after- initiation of UTT strategy had baseline WHO stage I. Ten (3.5\%) patients who started ART before and 11 (3.8\%) patients who started ART after UTT strategy had experienced opportunistic infections (Table 3).

\section{Proportion of ART Adherence}

The overall proportion of ART adherence using Morisky scale and self-report was $52.3 \%$ (95\% Cl: (48.4\%, $56.2 \%))$ and $95 \%$ (95\% Cl: $(93.5 \%, 96.8 \%))$ respectively. The proportion of ART adherence among patients who were enrolled before the initiation of UTT strategy using Morisky scale and patient self-reports was $55.4 \%$ (95\% Cl: $(49.9 \%, 60.6 \%))$ and $94.1 \%$ (95\% Cl: $(91 \%, 96.9 \%))$ respectively. The proportion of ART adherence among patients who were enrolled after initiation of UTT strategy using Morisky scale and patient self-report was49.3\% (95\% Cl: $(43.5 \%, 54.8 \%))$ and 95.9\% (95\% Cl: $(93.2 \%, 98.2 \%))$ respectively (Figure 1 and 2).

\section{Factors Associated with ART Adherence}

Adherence to ART measured by the Morisky scale was used to fit logistic regression model. Bi-variable and multivariable binary logistic regression analyses were done. The finding indicated that adult HIV patients who didn't experience depression were 3.9 times more likely to have better adherence than patients who experienced depression (AOR $=3.87,95 \% \mathrm{Cl}$ : $(1.96,7.64))$. Those adult HIV patients who had eaten three or more meals per day were 2.65 times more likely to have better adherence than patients who had eaten less than three meals per day $(A O R=2.65,95 \% \mathrm{Cl}:(1.08,6.49))$. Concomitant illness has also been significantly associated with ART adherence. Those patients without concomitant illness were $58 \%$ less likely to adhere their ART medication as compared to patients with concomitant illness (AOR $=0.42$, $95 \% \mathrm{Cl}:(0.23,0.76))($ Table 4$)$.

\section{Discussion}

We found that the overall proportion of ART adherence measured by using the Morisky scale (52.3\%) was lower than adherence measured by self-report (95\%). The proportion of adherence to ART didn't vary between those patients who started ART before the introduction of UTT strategy and patients who started 
ART after the introduction of UTT strategy. This study identified that depression, meal frequency and concomitant illness were factors associated with ART adherence.

This study revealed that the overall proportion of ART adherence measured by using the Morisky scale is lower than the proportion of ART adherence measured by using a seven-day patient self-report. The reason behind this difference could be self-report overestimate adherence since it is vulnerable to the dynamics of the provider-patient relationship and measurement biases like social desirability, recall and response bias (24). The overall proportion of ART adherence measured by using the Morisky scale is lower than the current recommended level of adherence by WHO (3). It is also lower than the proportion of ART adherence reported by a study conducted in Bale zone, Ethiopia(68.3\%) which was measured by using four items MMAS) (7). The reason for this difference could be originally the four items Morisky medication adherence scale had low sensitivity (81\%) and specificity (44\%) to detect ART adherence and had 0.61 Cronbach's alpha value, which is below the acceptable value of 0.7 . Whereas the modified eightitem MMAS had high sensitivity (93\%) and specificity (53\%) with 0.83 Cronbach's alpha value (25). Our finding is also lower than the proportion of ART adherence reported by a study conducted in India (78\%), measured with the same scale (23).

The overall proportion of ART adherence measured by using seven-day patient self-report is consistent with the current WHO recommendation (3) as well as with a study conducted in Debre Birhan Referral Hospital(10).

Both Morisky scale and patient self-reports results showed that the proportion of ART adherence is not varying between patients who were enrolled before initiation of UTT strategy and patients who were enrolled after initiation of UTT strategy. This might be due to the reason that those patients who were enrolled before initiation of UTT strategy stay a long time on pre-ART which helped them to have good knowledge about adherence while those patients who were enrolled after initiation of UTT strategy could also be exposed to different media, educational materials and took an extensive adherence counseling that lead them to have similar ART adherence. This study also revealed comparable knowledge of HIV/AIDS and its treatment between those patients who initiated ART before $(72.7 \%)$ and after $(64.7 \%)$ the initiation of UTT strategy.

In this study, absence of depression was appeared to be positively associated with good ART adherence. Similarly, other literature showed that absence of depression was positively associated with good ART adherence $(20,26,27)$. The possible reason for this might be that those patients who were not depressed might not experience hopelessness and demoralization which could in-turn prevent them skip or forget their regular treatment.

Meal frequency of three or more was also positively associated with good ART adherence. This finding is not in agreement with evidence obtained from Northern Ethiopia, in which daily eating pattern had no association with ART adherence(27). Though the causal mechanism is not clear yet, the association between reduced meal frequency and ART non-adherence might be because of perceived fear or actual experiences with increased hunger on ART when people have to take ART on an empty stomach. It might 
be also because of fear or actual experiences with exacerbated side effects of ART in the absence of adequate nutritional intake (28).

The absence of concomitant illness has been negatively associated with good ART adherence. Similarly, a study conducted in Bale zone, Ethiopia also revealed the absence of concomitant illness has been negatively associated with good ART adherence (7). This might be because of patients without concomitant illness reluctance to take their ART medication due to feeling well. On the other hand, patients with concomitant illness might adhere to their ART medication due to fear of death imposed by both HIV/AIDS and the concomitant illness. They might also stick to their ART medication because of having better information exposure about the importance of adhering ART medication as a result of double counseling service they might receive from different health care providers working for both HIV/AIDS and concomitant illness.

This study has strengths as well as limitations. As strengths, we used multiple ART adherence measurements. Adherence to ART was measured by patient self-report and the eight-item MMAS. In selfreported adherence, the last seven days ART adherence was used to minimize recall bias. As a limitation, adherence measured by the Morisky scale might be affected by recall bias as patients were asked to answer the questions by considering their medication intake since they start ART medication. Being a facility-based study can underestimate non-adherence because it is prone to miss defaulters and delayed visitors of their appointment.

\section{Conclusions}

In this study, the overall proportion of ART adherence measured using Morisky scale was low whereas the overall proportion of ART adherence measured using self-reports was consistent with the current WHO recommendation. Starting ART before or after initiation of the UTT strategy had no effect on ART adherence. Depression, meal frequency and concomitant illness were factors significantly associated with ART adherence. Patient counseling on adherence should target those patients without concomitant illness since they might not adhere to their treatment due to feeling well. In order to improve ART adherence, health care providers should screen patients on ART for depression and should provide appropriate and prompt medical as well as psychological treatment in collaboration with mental health professionals. Nutritional support with ART adherence counseling should be emphasized for those patients who are food insecured.

\section{List Of Abbreviations}

AIDS-Acquired Immune Deficiency Syndrome; AOR-Adjusted Odd Ratio; ART-Anti- Retroviral Therapy; BScBachelor of Science; $\mathrm{CD}_{4}$ _Cluster Differentiation 4; Cl-Confidence Interval; COR-Crude Odds Ratio; HIVHuman Immunodeficiency Virus;IQR-Inter Quartile Range; MMAS-Morisky Medication Adherence Scale; PHQ-9-Patient Health Questionnaire 9; SD-Standard Deviation; SE-Standard Error;SPSS-Statistical 
Package for Social Science; SSA-Sub Saharan Africa; UTT-Universal Test and Treat; VL- Viral Load; WHOWorld Health Organization.

\section{Declarations}

\section{Ethical Approval and Consent to Participate}

Ethical clearance was taken from the Ethical Review Committee of Wollo University College of Medicine and Health Sciences. Letter of permission to conduct the study was obtained from Administrative Health Office of Dessie town and each health facility administration. After explaining the purpose of the study, a verbal informed consent was obtained from participants before data collection. They were informed that participating in the study is voluntary and refusal to participate would not compromise the medical care they receive from the ART clinics. Privacy of the client and confidentiality of information they give was secured at all levels.

\section{Consent for Publication:}

Not applicable.

\section{Availability of Data and Material:}

The datasets used for the current study is available from the corresponding author on reasonable request.

\section{Competing interest:}

The authors declare that they have no competing interests.

\section{Financial Disclosure:}

Wollo University was funded the research. The funders had no role in study design, data collection and analysis, decision to publish, or preparation of the manuscript.

\section{Author's contribution}

$Y D$ : Initiated the research concept, wrote the research proposal, involved in the data collection processes, did data entry and analysis and wrote the manuscript. $D N$ : enriched the research concept and proposal development process and critically reviewed the manuscript. FT: involved in reviewing the research paper. $M Y$ : involved in reviewing the research paper. All the authors read and approved the final manuscript. 


\section{Acknowledgement}

We would like to express our deepest gratitude to Wollo University College of Medicine and Health Sciences Department of Public Health for providing financial and material support to conduct this research. We would like to acknowledge the City Administrative Health Office and ART clinics of Dessie town for providing necessary information to conduct the study. Finally, we would like to thank the study participants for their cooperation during the data collection process.

\section{References}

1. UNAID. Joint United Nations Program on HIV/AIDS, Data on HIV estmate; Geneva:UNAID, 2017.

2. Ethiopian Public Health Inistitute; HIV Related Estimates and Projections for Ethiopia. Addis Ababa; 2017.

3. WHO. scaling up HIV/AIDS priority intervention in the health sectors, progress report: towards universal access. www.who.int/hiv/mediacentre/universal_access_progress_report_en.pdf

4. Mills E, Nachega J, Buchan I. Adherence to Antiretroviral Therapy in Sub-Saharan Africa and North America. 2009.

5. Heestermans T, Browne JL, Aitken SC, Vervoort SC, Klipstein-Grobusch K: Determinants of adherence to antiretroviral therapy among HIV-positive adults in sub-Saharan Africa: a systematic review. BMJ Glob Health 2016, 1(4):e000125.

6. Abera A, Fenti B, Tesfaye T, Balcha F. Factors Influencing Adherence to Antiretroviral Therapy among People Living With HIV/AIDS at ART Clinic in Jimma University Teaching Hospital, Southwest. J Pharmacol. 2016;1(1):1-6.

7. Beshir M, Tesfaye A. Factors affecting adherence to anti-retroviral treatment among patients living with HIV / AIDS, in Bale zone, southeastern Ethiopia. 2017;9(March):67-73.

8. Abayneh W: Factors Associated With Level of Adherence to Antiretroviral Therapy in People Living with HIV/AIDS at Adama Hospital Medical College Art Clinic, Oromiya Regional State, Ethiopia. International Journal of HIV/AIDS Prevention, Education and Behavioural Science 2017, 3(3):22.

9. Belayihun B, Negus R: Antiretroviral Treatment Adherence Rate and Associated Factors among People Living with HIV in Dubti Hospital, Afar Regional State, East Ethiopia. Int Sch Res Notices 2015, 2015:187360.

10. Ketema AK, Shewangizaw Weret Z: Assessment of adherence to highly active antiretroviral therapy and associated factors among people living with HIV at Debrebrihan Referral Hospital and Health Center, Northeast Ethiopia: a cross-sectional study. HIV AIDS (AuckI) 2015, 7:75-81.

11. Enriquez M, McKinsey DS: Strategies to improve HIV treatment adherence in developed Ethiopian Federal Ministry of Health; Guidelines for Management of Opportunistic Infections and AntiRetroviral Treatment in Adolescents and Adults in Ethiopia, HIV prevention and control office; 2008. 
13. David R, Edwin D, Richard A. Non-adherence to highly active antiretroviral therapy predicts progression to AIDS. 2001.

14. Mannheimer S, Friedland G, Matts J,et al. The consistency of adherence to antiretroviral therapy predicts biologic outcomes for human immunodeficiency virus-infected persons in clinical trials. Clin Infect Dis. 2002;34(8):1115-21.

15. Doyore F, Moges B: Adherence to Antiretroviral Treatment Services and Associated Factors among Clients Attending ART Clinics in Hosanna Town, Southern Ethiopia. Journal of AIDS \& Clinical Research 2016, 7(7).

16. Reda AA, Biadgilign S: Determinants of Adherence to Antiretroviral Therapy among HIV-Infected Patients in Africa. AIDS Res Treat 2012, 2012:574656.

17. World Health Organization; Consolidated guidelines on the use of antiretroviral drugs for treating and preventing HIV infecion recommendations for a public health approach. Geneva: 2013.

18. World Health Organization; Guideline on when to start antiretroviral therapy and on pre-exposure prophylaxis for HIV; Geneva, 2015.

19. Dessie Town Administrative Health Office Report. January 2018.

20. Letta S, Demissie A, Oljira L, Dessie Y: Factors associated with adherence to Antiretroviral Therapy (ART) among adult people living with HIV and attending their clinical care, Eastern Ethiopia. BMC Int Health Hum Rights 2015, 15:33

21. Mthembu TG, Van Wyk B: Patients' knowledge and beliefs about antiretroviral treatment and factors associated with adherence in Mpumalanga Province, South Africa. Health SA Gesondheid 2014, 19(1).

22. Nikus N, Aman M, Brihnu Z. HIV stigma and associated factors among anti-retroviral treatment clients in Jimma town, Southwest Ethiopia. 2016;183-93.

23. Gokarn A, Narkhede M, Pardeshi G, Doibale M. Adherence to antiretroviral therapy. J Assoc Physicians India. 2012;60(December):16-20.

24. Shi L, Liu J, Fonseca v, et al; Correlation between adherence rates measured by MEMS and selfreported questionnaires: a meta-analysis. Health and Quality of Life Outcomes, 2010.

25. Tan X, et al. Review of the four item Morisky Medication Adherence Scale (MMAS-4) and eight item Morisky Medication Adherence Scale ( MMAS-8 ), 2014.

26. Alagaw A, Godana W, Taha M, Dejene T. Factors associated with antiretroviral treatment adherence among adult patients in Wolaita Soddo. 2014;2(2):69-77.

27. Gebrezgabher BB, Kebede Y, Kindie M, Tetemke D, Abay M, Gelaw YA: Determinants to antiretroviral treatment non-adherence among adult HIV/AIDS patients in northern Ethiopia. AIDS Res Ther2017, 14:16. 
28. Sera Y. et al, A review of the role of food insecurity in adherence to care and treatment among adult and pediatric populations living with HIV and AIDS, 2014;18(0 5): 505-515

\section{Tables}

Table1: Socio-demographic characteristics of before- and after-UTT ART initiators at Dessie town clinics, 2018.<.p> 
Sex

Male

$118(40.8 \%)$

$140(47.9 \%)$

Female

$171(59.2 \%)$

$152(52.1 \%)$

Marital status

Single

$42(14.5 \%)$

$45(15.4 \%)$

Married

$155(53.6 \%)$

$178(61.0 \%)$

widowed

$29(10 \%)$

$13(4.5 \%)$

Divorced

$63(21.8 \%)$

$56(19.2 \%)$

Ethnicity

Amhara

$262(90.7 \%)$

275 (94.2\%)

Others

$27(9.3 \%)$

17 (5.8\%)

\section{Religion}

Christian $^{* *}$

129 (44.6\%)

102 (34.9\%)

Muslim

$160(55.4 \%)$

$190(65.1 \%)$

Residence

Urban

$232(80.3 \%)$

$218(74.7 \%)$

Rural

57 (19.7\%)

$74(25.3 \%)$

Level of education

No formal education

$67(23.2 \%)$

$71(24.3 \%)$

Primary education

$112(38.8 \%)$

116 (39.7\%)

Secondary education

$71(24.6 \%)$

$78(267 \%)$

College and above

39 (13.5\%)

$27(9.2 \%)$

\section{Occupation}

Government employee

Private employee

39 (13.5\%)

$31(10.6 \%)$

House wife

45 (15.6\%)

$36(12.3 \%)$

Merchant

$35(12.1 \%)$

$52(17.8 \%)$

Farmer

$54(18.7 \%)$

$55(18.8 \%)$

Daily laborer

48 (16.6\%)

$62(21.2 \%)$

49 (17\%)

$39(13.4 \%)$ 

Others $^{* * *}$
$19(6.6 \%)$
$17(5.8 \%)$

Family size

$$
\begin{array}{lll}
\leq 5 & 269(93.1 \%) & 277(94.9 \%) \\
\geq 6 & 20(6.9 \%) & 15(5.1 \%)
\end{array}
$$

Frequency of meal/day

$\begin{array}{lll}<3 \text { meal/day } & 21(7.3 \%) & 8(2.7 \%) \\ \geq 3 \text { meal/day } & 268(92.7 \%) & 284(97.3 \%)\end{array}$

With whom patient live

Family

Partner

Alone

Wealth quintile

Lowest

Lower

Middle

Higher

Highest
$113(39.1 \%)$

$140(48.4 \%)$

$36(12.5 \%)$

$59(20.4 \%)$

$72(25.0 \%)$

$26(9.0 \%)$

$87(30.1 \%)$

$45(15.5 \%)$
77 (26.4\%)

$179(61.3 \%)$

$36(12.3 \%)$

$50(17.0 \%)$

87 (29.8\%)

$32(11.0 \%)$

$62(21.2 \%)$

$49(21.0 \%)$

* Oromo and Tigray, ${ }^{* *}$; protestant and orthodox tewahido, ${ }^{* * *}$ student and jobless, UTT universal test and treat strategy, ART anti-retroviral therapy

Table2: Psychosocial and behavioral characteristics of before- and after-UTT ART initiators at Dessie town clinics, 2018. 


\section{Variables}

Before

After

$\mathrm{UTT}(\mathrm{n}=289)$

$\mathrm{UTT}(\mathrm{n}=292)$

\section{Using reminders}

Yes

$278(96.2 \%)$

$285(97.6 \%)$

No

$11(3.8 \%)$

$7(2.4 \%)$

Feel comfort while taking medication in front of others

Yes

$230(79.6 \%)$

$212(72.6 \%)$

No

59 (20.4\%)

$80(27.4 \%)$

Taking adherence counseling

Yes

$100(100 \%)$

291 (99.7\%)

No

$0(0 \%)$

$1(0.3 \%)$

Disclosure status

Yes

$264(91.3 \%)$

$270(92.5 \%)$

No

$25(8.7 \%)$

$22(7.5 \%)$

Getting support

Yes

$51(17.6 \%)$

$21(7.2 \%)$

No

$238(82.4 \%)$

$271(92.8 \%)$

Taking alcohol

Yes

$69(23.9 \%)$

$34(11.6 \%)$

No

$220(76.1 \%)$

$258(88.4 \%)$

Smoking cigarette

Yes

$8(2.8 \%)$

7 (2.4\%)

No

$281(97.2 \%)$

$285(97.6 \%)$

Chewing chat

Yes

$19(6.6 \%)$

$17(5.8 \%)$

No

$270(93.4 \%)$

$275(94.2 \%)$

Having multiple sexual partner

Yes

$29(10 \%)$

$36(12.3 \%)$

No

$260(90 \%)$

$256(87.7 \%)$ 


\section{Knowledge to ART}

Good

Poor

\section{Stigma}

Yes

No

\section{Depression}

Yes

No

UTT universal test and treat strategy, ART anti-retroviral theraphy

Table 3: Disease related characteristics of before- and after-UTT ART initiators at Dessie town clinics, 2018.
$210(72.7 \%) \quad 189(64.7 \%)$

$79(27.3 \%) \quad 103(35.3 \%)$

$87(30.1 \%) \quad 84(28.8 \%)$

$202(69.9 \%) \quad 208(71.2 \%)$

$40(13.8 \%) \quad 35(12 \%)$

$249(86.2 \%) \quad 257(88 \%)$ 


\begin{tabular}{|c|c|c|}
\hline Variables & Before UTT(n=289) & After UTT $(n=292)$ \\
\hline \multicolumn{3}{|c|}{ Baseline CD4 count } \\
\hline$<200$ & $156(54 \%)$ & $65(22.3 \%)$ \\
\hline $200-350$ & $112(38.8 \%)$ & 92 (31.5\%) \\
\hline $351-500$ & $14(4.8 \%)$ & $84(28.8 \%)$ \\
\hline$>500$ & 7 (2.4\%) & $51(17.5 \%)$ \\
\hline \multicolumn{3}{|c|}{ Recent CD4 count } \\
\hline$<200$ & $22(7.6 \%)$ & $44(15.1 \%)$ \\
\hline $200-350$ & $67(23.2 \%)$ & $88(30.1 \%)$ \\
\hline $351-500$ & $84(29.1 \%)$ & 87 (29.8\%) \\
\hline$>500$ & $116(40.1 \%)$ & $73(25 \%)$ \\
\hline \multicolumn{3}{|c|}{ Baseline WHO stage } \\
\hline $\mathrm{I}$ & $44(15.2 \%)$ & 237 (81.2\%) \\
\hline II & $92(31.8 \%)$ & 33 (11.3\%) \\
\hline III & $123(42.6 \%)$ & $14(4.8 \%)$ \\
\hline IV & $30(10.4 \%)$ & $8(2.7 \%)$ \\
\hline \multicolumn{3}{|c|}{ Current WHO stage } \\
\hline $\mathrm{T} 1$ & 277 (95.8\%) & $279(95.5 \%)$ \\
\hline$\geq \mathrm{T} 2$ & $12(4.2 \%)$ & $13(4.5 \%)$ \\
\hline \multicolumn{3}{|c|}{ Current viral load } \\
\hline Not done & $0(0.0 \%)$ & $110(37.7 \%)$ \\
\hline$<1000$ & 247 (85.5\%) & $156(53.4 \%)$ \\
\hline$\geq 1000$ & $42(14.5 \%)$ & $26(8.9 \%)$ \\
\hline \multicolumn{3}{|c|}{ Concomitant diseases } \\
\hline Yes & $59(20.4 \%)$ & 34 (11.6\%) \\
\hline No & 230 (79.6\%) & $258(88.4 \%)$ \\
\hline \multicolumn{3}{|c|}{ Opportunistic diseases } \\
\hline Yes & $10(3.5 \%)$ & $11(3.8 \%)$ \\
\hline No & 279 (96.5\%) & $281(96.2 \%)$ \\
\hline
\end{tabular}


UTT universal test and treat strategy, ART anti-retroviral therapy

Table 4: Factors associated with ART adherence among before- and after-UTT ART initiators at Dessie town clinics, 2018. 


\begin{tabular}{|c|c|c|c|c|c|}
\hline \multirow[t]{2}{*}{ Variables } & & \multicolumn{2}{|c|}{ Adherence $(n=581)$} & \multirow[t]{2}{*}{ COR(95\%CI) } & \multirow[t]{2}{*}{ AOR(95\%CI) } \\
\hline & & Good & Poor & & \\
\hline \multirow[t]{2}{*}{ Age } & & 304 & 277 & $1.034(1.02$ & 1.01(0.98, \\
\hline & & $(52.3 \%)$ & $(47.7 \%)$ & $1.05)^{* * *}$ & $1.04)$ \\
\hline \multirow[t]{7}{*}{ Marital status } & Single & $28(9.2 \%)$ & $59(21.3 \%)$ & 1 & 1 \\
\hline & Married & 181 & 152 & $2.51(1.52$ & $1.53(0.78$ \\
\hline & & $(59.5 \%)$ & $(54.9 \%)$ & $4.13)^{* * *}$ & 3.03) \\
\hline & widowed & $28(9.2 \%)$ & $14(5.1 \%)$ & 4.21 & $2.34(0.82$ \\
\hline & & & & $9.23)^{* * *}$ & $6.66)$ \\
\hline & Divorced & $67(22 \%)$ & $52(18.8 \%)$ & $2.72(1.52$ & $2.02(0.97$ \\
\hline & & & & $4.84)^{* * *}$ & 4.19) \\
\hline Frequency of meal & $<3$ & $11(3.6 \%)$ & $18(6.5)$ & 1 & 1 \\
\hline \multirow[t]{2}{*}{ /day } & $\geq 3$ & 293 & $259(93.5 \%)$ & $1.85(0.86$ & $2.65(1.08$ \\
\hline & & $(96.4 \%)$ & & 3.99) & $6.49)^{*}$ \\
\hline \multirow[t]{4}{*}{ Chewing chat } & Yes & $15(4.9 \%)$ & $21(7.6 \%)$ & $1.58(0.80$ & 0.89 (0.37, \\
\hline & & & & 3.13) & 2.19) \\
\hline & No & 289 & 256 & 1 & 1 \\
\hline & & $(95.1 \%)$ & $(92.4 \%)$ & & \\
\hline \multirow[t]{4}{*}{ Taking alcohol } & Yes & 41 & $62(22.4 \%)$ & 1 & 1 \\
\hline & & $(13.5 \%)$ & & & \\
\hline & No & 263 & 215 & 1.85 (1.20, & $1.44(0.84$ \\
\hline & & $(86.5 \%)$ & $(77.6 \%)$ & $2.85)^{* *}$ & $2.48)$ \\
\hline Multiple sexual & Yes & 18 (5.9\%) & 47 (17\%) & 1 & 1 \\
\hline \multirow[t]{2}{*}{ partner } & No & 286 & 230 (83\%) & 3.25 (1.84, & $1.56(0.78$ \\
\hline & & $(94.1 \%)$ & & $5.74)^{* * *}$ & $3.10)$ \\
\hline \multirow[t]{3}{*}{ Depression } & Yes & $16(5.3 \%)$ & 59 (21.3\%) & 1 & 1 \\
\hline & No & 288 & 218 & 4.87 (2.73, & 3.87 (1.96, \\
\hline & & $(94.7 \%)$ & (78.7\%) & $8.70)^{* * *}$ & $7.64)^{* * *}$ \\
\hline \multirow[t]{2}{*}{ Stigma } & Yes & 64 & 107 & 1 & 1 \\
\hline & & $(21.1 \%)$ & (38.6\%) & & \\
\hline
\end{tabular}




\begin{tabular}{|c|c|c|c|c|c|}
\hline & No & $\begin{array}{l}240 \\
(78.9 \%)\end{array}$ & $\begin{array}{l}170 \\
(61.4 \%)\end{array}$ & $\begin{array}{l}2.36(1.64, \\
3.41)^{* * *}\end{array}$ & $\begin{array}{l}1.31(0.60, \\
2.89\end{array}$ \\
\hline \multirow[t]{2}{*}{ Follow appointment } & Yes & $\begin{array}{l}223 \\
(73.4 \%)\end{array}$ & $\begin{array}{l}172 \\
(62.1 \%)\end{array}$ & $\begin{array}{l}1.68(1.18, \\
2.39)^{* *}\end{array}$ & $\begin{array}{l}0.66,(0.43, \\
2.93)\end{array}$ \\
\hline & No & $\begin{array}{l}81 \\
(26.6 \%)\end{array}$ & $\begin{array}{l}105 \\
(37.9 \%)\end{array}$ & 1 & 1 \\
\hline \multirow[t]{3}{*}{$\begin{array}{l}\text { Number of } \\
\text { tablet/day }\end{array}$} & 1 & $\begin{array}{l}159 \\
(52.3 \%)\end{array}$ & $\begin{array}{l}107 \\
(38.6 \%)\end{array}$ & $\begin{array}{l}1.91(1.32, \\
2.78)^{* * *}\end{array}$ & $\begin{array}{l}3.19(0.10, \\
104.62)\end{array}$ \\
\hline & 2 & $\begin{array}{l}58 \\
(19.1 \%)\end{array}$ & $58(20.9 \%)$ & $\begin{array}{l}1.29(0.81, \\
2.04)\end{array}$ & $\begin{array}{l}0.73(0.12, \\
4.49)\end{array}$ \\
\hline & 3 & $\begin{array}{l}87 \\
(28.6 \%)\end{array}$ & $\begin{array}{l}112 \\
(40.4 \%)\end{array}$ & 1 & 1 \\
\hline \multirow[t]{2}{*}{$\begin{array}{l}\text { Frequency of } \\
\text { dosage }\end{array}$} & Once & $\begin{array}{l}170 \\
(55.9 \%)\end{array}$ & $122(44 \%)$ & $\begin{array}{l}1.61(1.16, \\
2.24)^{* *}\end{array}$ & $\begin{array}{l}1.61(0.24 \\
10.65)\end{array}$ \\
\hline & Twice & $\begin{array}{l}134 \\
(44.1 \%)\end{array}$ & 155 (56\%) & 1 & 1 \\
\hline \multirow[t]{2}{*}{ Enrollment status } & AUTT & $\begin{array}{l}144 \\
(47.4 \%)\end{array}$ & $\begin{array}{l}148 \\
(53.4 \%)\end{array}$ & 1 & 1 \\
\hline & BUTT & $\begin{array}{l}160 \\
(52.6 \%)\end{array}$ & $\begin{array}{l}129 \\
(46.6 \%)\end{array}$ & $\begin{array}{l}1.28(0.92, \\
1.77)\end{array}$ & $\begin{array}{l}1.49(0.97 \\
2.29)\end{array}$ \\
\hline \multirow[t]{4}{*}{ Current regimen } & $\mathrm{a}$ & $\begin{array}{l}158 \\
(52 \%)\end{array}$ & 108 (39\%) & 1 & 1 \\
\hline & $\mathrm{b}$ & $\begin{array}{l}50 \\
(16.4 \%)\end{array}$ & $71(25.6 \%)$ & $\begin{array}{l}0.48(0.31 \\
0.75)^{* * *}\end{array}$ & $\begin{array}{l}2.08(0.16 \\
26.51)\end{array}$ \\
\hline & $\mathrm{C}$ & $\begin{array}{l}48 \\
(15.8 \%)\end{array}$ & $42(15.2 \%)$ & $\begin{array}{l}0.78(0.48, \\
1.26)\end{array}$ & $\begin{array}{l}3.57(0.24 \\
53.7)\end{array}$ \\
\hline & Others $^{\circledR}$ & $\begin{array}{l}48 \\
(15.8 \%)\end{array}$ & $56(20.2 \%)$ & $\begin{array}{l}0.59(0.37 \\
0.93)^{*}\end{array}$ & $\begin{array}{l}2.52(0.20 \\
32.43)\end{array}$ \\
\hline $\begin{array}{l}\text { Taking other } \\
\text { medication }\end{array}$ & Yes & $\begin{array}{l}124 \\
(40.8 \%)\end{array}$ & $\begin{array}{l}151 \\
(54.5 \%)\end{array}$ & 1 & 1 \\
\hline
\end{tabular}




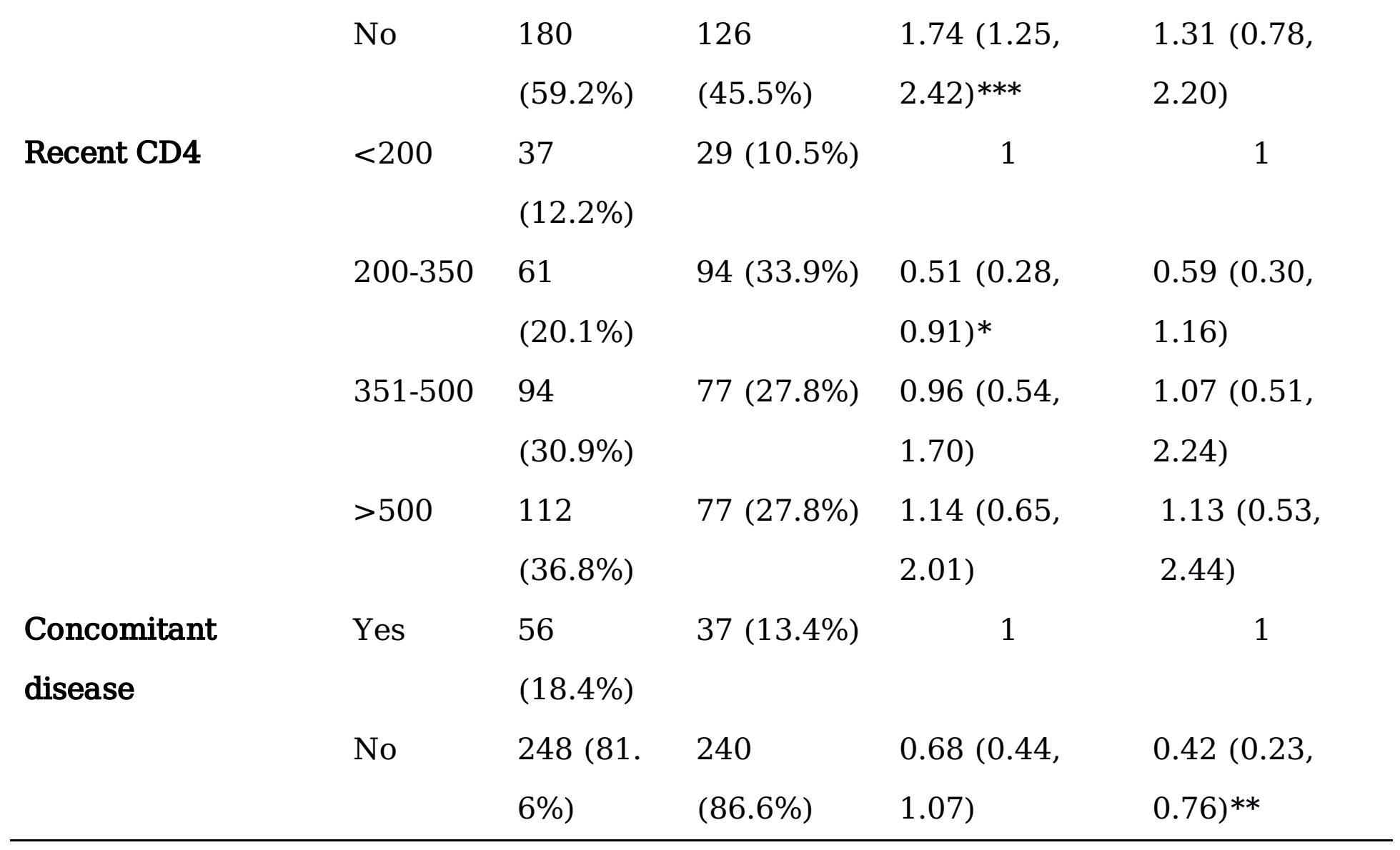

COR Crude Odds Ratio; AOR Adjusted Odds Ratio; AUTT after universal test and treat strategy; BUTT before universal test and treat strategy; ART anti-retroviral therapy; *Significant at $\mathrm{P}<0.05$; $* *$ significant at $\mathrm{P}<0.01$; *** significant at $\mathrm{P} \leq 0.001$ in the bivariable and multivariable logistic regression analysis; ${ }^{\odot} \mathrm{AZT}-3 \mathrm{TC}-\mathrm{EFV}$, TDF-3TC-ATVr, AZT-3TC-ATVr, ABC-3TCLPVr and AZT-3TC-LPVr; a TDF-3TC-EFV; b TDF-3TC-NVP; c AZT-3TC-NVP

\section{Figures}

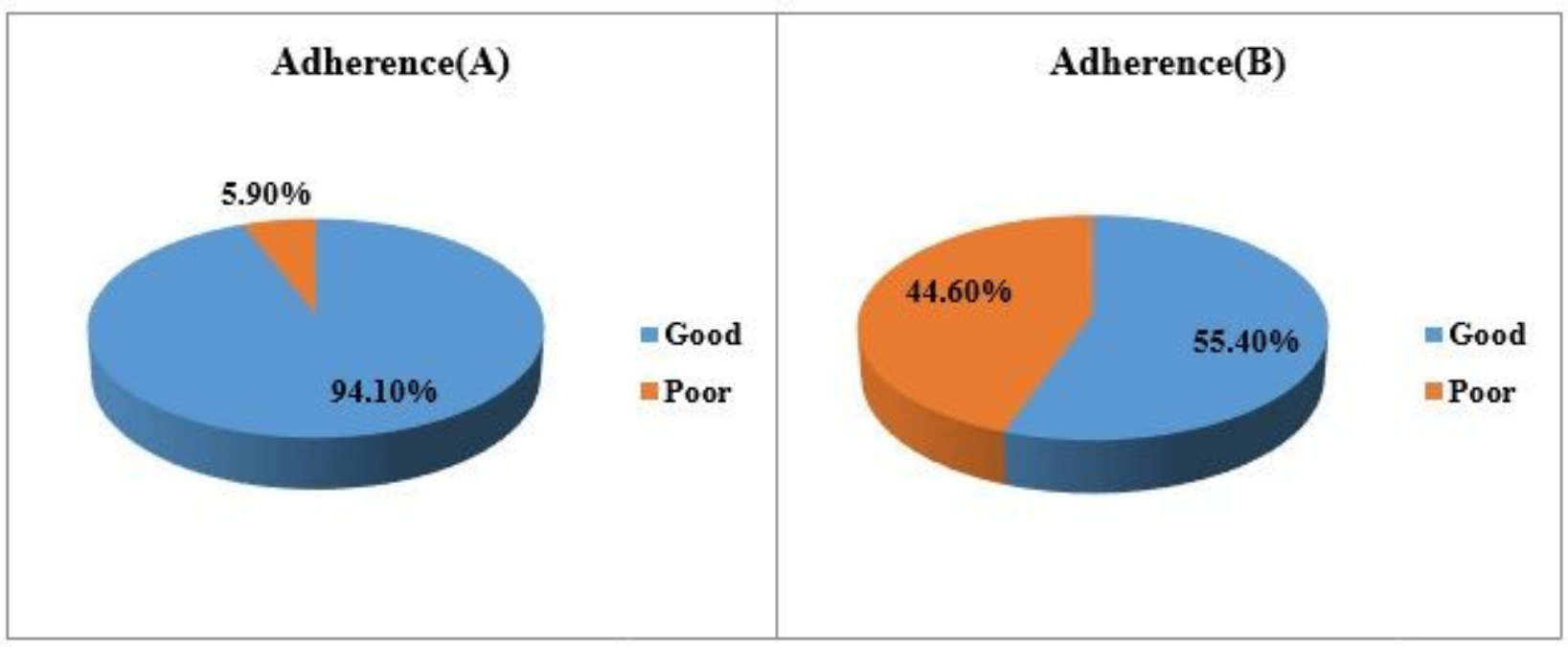


Figure 1

ART adherence using self-report (A) and Morisky scale(B) among before-UTT ART initiators at Dessie town clinics, 2018. Legend: Figure 1A, in the left side, indicates the proportion of ART adherence measured using patient self-report of last seven days adherence to ART medication while figure 1B, in the right side, indicates the proportion of ART adherence measured using the Morisky Medication Adherence Scale for Adult HIV positive patients who initiated the ART before the launch of universal test and treat (UTT) strategy.

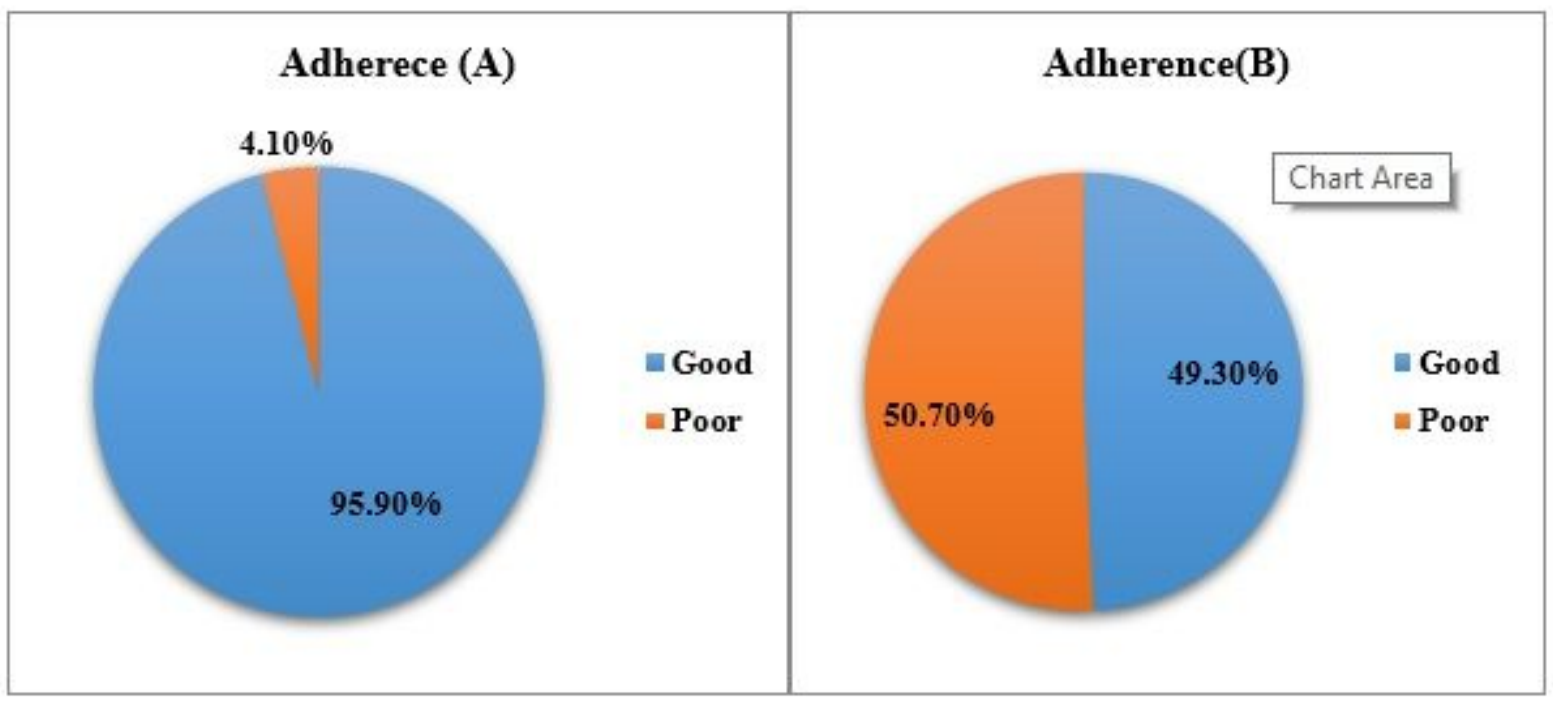

\section{Figure 2}

ART adherence using self-report (A) and Morisky scale(B) amongafter-UTT ART initiators at Dessie town clinics, 2018. Legend: Figure 2A, in the left side, indicates the proportion of ART adherence measured using patient self-report of last seven days adherence to ART medication while figure $2 B$, in the right side, indicates the proportion of ART adherence measured using the Morisky Medication Adherence Scale for HIV positive adult patients who initiated the ART after the launch of universal test and treat (UTT) strategy. 